\title{
« Danses macabres »: Une technologie culturelle du massacre des Tutsi au Rwanda
}

"Dance of Death": Cultural Technology of Tutsi Slaughter in Rwanda

Thomas Riot, Nicolas Bancel et Herrade Boistelle

\section{OpenEdition \\ 12 Journals}

\section{Édition électronique}

URL : http://journals.openedition.org/conflits/19403

DOI : 10.4000/conflits.19403

ISSN : $1777-5345$

Éditeur :

CCLS - Centre d'études sur les conflits lilberté et sécurité, L'Harmattan

\section{Édition imprimée}

Date de publication : 20 décembre 2016

Pagination : 169-186

ISBN : 978-2-343-11087-5

ISSN : 1157-996X

Référence électronique

Thomas Riot, Nicolas Bancel et Herrade Boistelle, « « Danses macabres » : Une technologie culturelle du massacre des Tutsi au Rwanda », Cultures \& Conflits [En ligne], 103-104 | automne/hiver 2016, mis en ligne le 20 décembre 2018, consulté le 30 mars 2021. URL : http://journals.openedition.org/ conflits/19403; DOI : https://doi.org/10.4000/conflits.19403 


\section{«Danses macabres» Une technologie culturelle du massacre des Tutsi au Rwanda}

\section{Thomas RIOT, Nicolas BANCEL et Herrade BOISTELLE}

Thomas Riot est chercheur FNS à la Faculté des Sciences sociales et politiques de l'Université de Lausanne. Après avoir réalisé une thèse traitant de la participation des activités physiques et sportives au processus de décolonisation $d u$ Rwanda, il s'intéresse aux cultures motrices et aux liens qu'elles entretiennent avec le nationalisme et la violence armée en Afrique.

Nicolas Bancel est historien, spécialiste de l'histoire coloniale et postcoloniale, professeur à la Faculté des Sciences sociales et politiques de l'Université de Lausanne. Il s'intéresse en particulier à l'histoire des mouvements de jeunesse, à l'anthropologie des représentations du corps et aux imaginaires politiques de la colonisation.

Herrade Boistelle est doctorante à la Faculté des Sciences sociales et politiques de l'Université de Lausanne. Dans le cadre de sa thèse, elle s'intéresse aux pratiques physiques et à leurs impacts sur la formation des jeunesses scolaires et militaires en Ouganda.

Kigali, le $1^{\text {er }}$ juillet 1987 . En ce jour de célébration du vingt-cinquième Lanniversaire de l'indépendance du Rwanda, un groupe de danseurs intore (élus, choisis) réalise une performance chorégraphique. Le spectacle prend forme au stade national de Kigali, érigé en scène commémorative de l'indépendance. Une troupe composée d'une centaine d'enfants et d'adultes spécialement sélectionnés pour l'occasion se place sur dix rangées parallèles au front de scène. L'alignement des intore se réalise par la constitution d'une série de rangs, selon un ordre chorégraphique qui se lie peu à peu au chant qui l'accompagne :

« Tu as dit adieu au pouvoir monarchique, le joug féodal et colonial sont partis ensemble, tu as obtenu la démocratie dont tu es fier [...] Cher Rwandais, jette un regard en arrière et souviens-toi du fouet, 
des travaux forcés [...] Souviens-toi des journées de marche où tu transportais des cadeaux au chef et au roi, en privant ta famille qui avait pourtant besoin, et une fois à destination tu n'obtenais même pas de récompense [...]. Viens qu'on fête les bienfaits de l'indépendance $\left[\right.$ paroles chantées] ${ }^{1} »$.

La marche d'approche se compose de pas esquissés, de flexions de genoux à peine marquées et de légères détentes de bras armés d'arcs et de lances. Cette entrée en matière est suivie du « cœur » de la chorégraphie. Au centre du groupe, un noyau de danseurs d'excellence (le «nombril ») est chargé de produire les "pas de base ", à partir desquels les autres danseurs (à gauche et à droite) règlent leur prestation. Partant de cette «base », chaque intore a la possibilité de broder des figures inédites plus ou moins complexes. Ils jouent avec les contre-temps, les objets (armes, coiffes, grelots, etc.) et les techniques soumises à l'ordre guerrier de la danse : saltations, retraits, feintes, attaques, etc. Il s'agit de composer un ensemble d'exhibitions en solos et en duos, après quoi chaque danseur est invité à rejoindre l'ensemble de la « mêlée » ainsi reconstituée. La troupe effectue alors une dernière et courte séquence suite à laquelle les intore quittent la scène en pas cadencés.

Ainsi, et tandis que les techniques des danseurs marquent les figures individuelles et collectives de la chorégraphie, la cérémonie du vingt-cinquième anniversaire de l'indépendance y ajoute un chant de danse qui pose la ligne idéologique de l'événement. Cette « ligne » s'inscrit dans l'activation du paradigme de la « révolution hutu », qui aurait conduit la libération du «peuple hutu » vis à vis de son oppresseur le plus direct : la monarchie tutsi.

Identifiée à un joug ancestral opposant deux catégories de la population rwandaise (serfs hutu vs féodaux tutsi), la féodalisation de la monarchie tutsi est en réalité un produit de l'histoire coloniale du Rwanda : le colonisateur (allemand de 1897 à 1916, belge de 1916 à 1962) délégua aux chefs tutsi l'œuvre d'exploitation de la majorité colonisée (identifiée à l'ethnie hutu), tout en essentialisant les rapports socio-ethniques en distinguant nettement, sur les cartes d'identité, l'appartenance ethnique. Être « hutu » signifiait être cantonné dans les fractions les plus pauvres et les emplois les moins dotés de l'économie coloniale. Au cours des années 1950, ce mode « autochtone » de domination coloniale favorisa la levée d'une « contre-élite hutu » 2, pour qui la décolonisation s'articulait à la disparition des privilèges attribués aux Tutsi. Entre 1959 et 1961, une série d'assassinats et de pogroms visant les élites tutsi précéda la chute de la monarchie Nyiginya et l'instauration de la Première

1. Celui-ci a été écrit et prononcé à l'occasion de la commémoration des 25 ans de l'indépendance, le 1er juillet 1987. Il fut retranscrit par Chrétien J.-P. (dir.), Rwanda. Les médias du génocide, Paris, Karthala, 1995, p. 121.

2. L'expression est de Ian Linden. Lire à ce sujet Linden I., Christianisme et pouvoir au Rwanda (1900-1990), Paris, Karthala, 1999. 
république hutu (le $1^{\mathrm{er}}$ juillet 1961) ${ }^{3}$. Après l'indépendance, les régimes successifs de Grégoire Kayibanda (1961-1973) et de Juvénal Habyarimana (19731994) renforcèrent cette ligne idéologique, continument mobilisée dans les appels à l'élimination des ennemis du « peuple majoritaire » ${ }^{4}$.

À la fin des années 1980, l'État rwandais - gouverné par le parti unique du Mouvement révolutionnaire national pour le développement (MRND) - est sujet à de nombreuses tensions politiques. Arrivé au pouvoir suite à un coup d'État (en 1973), le gouvernement Habyarimana se montre directement affecté par l'angoisse de voir le pays reconquis par des groupes ayant fui le pays entre 1959 et 1961, composés de Tutsi traqués par des groupes de radicaux hutu alliés à l'ancien gouvernement colonial. À la fin des années 1950, le colonisateur belge avait en effet opéré un retournement d'alliance en faveur de ces groupes hutu, retournement motivé par les aspirations de plus en plus nettement exprimées par les élites tutsi à conquérir l'indépendance. Dans les décennies qui suivent celle-ci, une diaspora composée de tutsi ayant fui les persécutions construisent un appareil militaire, le FPR (Front patriote rwandais), engagé dans la reconquête des territoires rwandais frontaliers avec le Burundi et l'Ouganda et, à plus long terme, visant la reconquête du pouvoir au Rwanda. Dans ce contexte, les bourgmestres et préfets du MRND puisent au jour le jour des informations relatives à l'activité de colonnes du FPR. À chaque nouvelle tentative d'incursion des troupes de la diaspora tutsi, chaque préfecture concernée déploie (à l'encontre des Tutsi de la zone) une violence physique révélatrice du sentiment d'angoisse paranoïaque qu'elle manifeste. Une archive missionnaire nous rappelle que ce mode extrême de réaction s'enracina au Rwanda au début des années 1960 :

«La réaction populaire a été très vive surtout dans les régions où les infiltrations avaient été plus nombreuses. La politique de la main tendue qui avait été celle du gouvernement, admettant dans ces services de nombreux Tutsi qui se disaient fidèles au nouveau régime, a été soldée par un échec. Chacun de ces employés versait un tiers de son traitement à l'UNAR (Union nationale rwandaise) pour acheter des armes qui devaient servir à reconquérir le pays et y réinstaller la monarchie. [...] Le Rwanda s'est retrouvé du jour au lendemain en état de guerre et les ennemis étaient partout. Évidemment on en a trouvé là où il n'y en avait pas, et ce fut l'occasion d'assouvir des vengeances personnelles. On ne peut nier qu'il y ait eu des massacres, et beaucoup trop ${ }^{5 » \text {. }}$

3. Pour une analyse socio-historique de ce basculement politique, lire Lemarchand R., Rwanda and Burundi, London, Pall Mall Press, 1970.

4. Du point de vue des identités politiques engagées dans le génocide, lire Hintjens H. M., "When identity becomes a knife: reflecting on the genocide in Rwanda ", Ethnicities, vol.1, $\mathrm{n}^{\circ} 1,2001$, pp. 25-55.

5. Lettre du Père Boutry au Père de Rasilly, Kabgayi, le 30 janvier 1964. Archives de la société des missionnaires d'Afrique (ARCHPB), Rome, Kabgayi 738 (1). 


\section{Une approche pratique et symbolique de la violence de masse}

Plus de vingt années après sa perpétration, le génocide qui a visé les Tutsi du Rwanda au cours de l'année 1994 a rarement été appréhendé dans le « comment » de son exécution ${ }^{6}$. Avec une très grande efficacité, des hommes « d'action », principaux acteurs des pogroms, ont mis en œuvre une technologie du massacre des Tutsi. Un premier groupe (majoritairement masculin et bien entraîné) avait fait ses classes dans les organisations de jeunesse et les cellules « d'animation » 7 du parti unique, le Mouvement révolutionnaire national pour le développement MRND 8 (jusqu'en 1991). Dès le début des années 1990, de nombreux cadres du parti s'engagèrent dans la formation d'un second groupe de miliciens ${ }^{9}$, le plus efficace exécutant des crimes. En effet, ensemble (cadres et exécutants), ces deux groupes forment la masse des 50000 hommes, femmes et jeunes (soit environ $25 \%$ des 200000 auteurs des tueries) et sont responsables du massacre d'environ 600000 Tutsi, soit environ $75 \%$ des 800000 meurtres exécutés en cent jours ${ }^{10}$.

Le massacre des Tutsi au Rwanda a fait l'objet de nombreuses publications. Tandis que l'essentiel de ces études se concentrent sur l'histoire sociopolitique ${ }^{11}$ et idéologique ${ }^{12}$ de l'événement, d'autres recherches l'abordent sur un plan micro-sociologique qui éclaire les dynamiques locales du mûrissement et de l'exécution des pogroms ${ }^{13}$. Ces études constituent le point de départ de notre approche de la violence de masse. Elles permettent d'une part

6. Claudine Vidal avait signalé cette «lacune » à peine quelques années après l'exécution du génocide : Vidal C., «Le génocide des Rwandais tutsi et l'usage public de l'histoire », Cahiers d'études africaines, vol. 38, n¹50-152, 1998, pp. 653-663.

7. L' " animation » correspond au développement de cellules politico-culturelles attachées aux communes et aux préfectures. Jeunes et adultes y pratiquaient danses, sports, débats, chansons...

8. Le MRND fut créé à la suite du coup d'État mené par les troupes du général Habyarimana en 1973.

9. Notamment les miliciens interahamwe, bien souvent prélevés dans la société civile.

10. Les chiffres que nous mentionnons ont été collectés par Scott Straus au cours de sa longue et minutieuse enquête sur «l'ordre du génocide » : Straus S., The order of genocide. Race, power and war in Rwanda, Ithaca/Londres, Cornell University Press, 2006. Les espaces de voisinage dans lesquels ont opéré les groupes de génocidaires soulignent la proximité et la localisation précise des attaques. Voir à ce sujet l'enquête menée par Dumas H., Le génocide au village. Le massacre des Tutsi au Rwanda, Paris, Éditions du Seuil, 2014.

11. La liste serait longue, mais il nous semble important de citer un auteur qui s'est penché sur les dimensions populaires du massacre : Kimonyo J-P., Rwanda. Un génocide populaire, Karthala, Paris, 2008.

12. Citons à ce sujet le travail de référence de Jean-Pierre Chrétien et ses collaborateurs, paru une année après l'exécution du génocide : Chrétien J-P. (dir.), Rwanda : les médias du génocide, op. cit.

13. Luft A., «Toward a dynamic theory of action at the micro-level of genocide: killing, desistance, and saving in 1994 Rwanda ", Sociological Theory, vol. 33, n², 2015, pp. 148-172 ; McDoom O. S., "Who killed in Rwanda genocide? Micro-space, social influence and individual participation in intergroup violence ", Journal of Peace Research, vol. 50, n4, 2013, pp. 453-467 ; Viret E., "La langue amère des temps nouveaux. Dynamiques de la violence au Rwanda rural ", Questions de recherche, n²9, août 2009; Dumas H., Le génocide au village, op. cit. 
de repérer les dispositifs (communaux, ruraux et nationaux) et les organisations qui se trouvent à l'origine des mutations sociales, économiques et politiques des acteurs de la violence. Elles autorisent d'autre part l'élaboration d'un savoir axé sur une démarche processuelle et générative : comment un certain nombre de relations sociales, de confrontations symboliques et de techniques de gouvernement interagissent dans l'engagement politique et armé des individus formés par ces dispositifs ?

Devenue célèbre à l'échelle internationale, la danse guerrière rwandaise (umuhamirizo) n'a jamais été interrogée sous l'angle de sa potentielle articulation aux politiques de la violence de masse. Comme d'autres «techniques réflexives du corps » ${ }^{14}$, la chorégraphie peut s'envisager selon les modifications corporelles entraînées par l'action des danseurs, ainsi que selon l'agencement politique et symbolique de leurs figures. En langue kinyarwanda, cette action se rapporte au verbe guhamiriza, qui désigne un dispositif corporel et guerrier destiné à «faire fuir l'ennemi de sorte que celui-ci prenne ses jambes à son cou sans oser même se retourner »15. De la fin des années 1970 au début des années 1990, de nombreux bourgmestres et animateurs locaux du MRND ont organisé le recrutement et l'initiation de plusieurs milliers de danseurs. On les retrouve à Kigali, à Kibemge ou à Gitesi, comme dans les 70 communes (sur 145) orchestratrices du mouvement : des groupes composés d'individus majoritairement issus de la communauté racialisée des Hutu, qui furent affectés - de gré ou de force - à la « technologie politique » des danses communales et nationales imibamirizo ${ }^{16}$ (dont l'action gubamiriza dérive).

Dans le cadre de nos réflexions sur la potentialité d'un lien entre ces techniques et l'exercice de la violence de masse, nous proposons d'étudier les actions menées par des corps sur d'autres corps : le « corps propre », celui que l'on peut toucher, combiné à la "chair », c'est-à-dire les dimensions éprouvées de la corporéité. À ce sujet, notons que les «corps dansants » qui se trouvent au centre de notre propos ne constituent pas des instruments « prêts à tuer ». Suivant le registre conceptuel foucaldien de la « microphysique du pouvoir ", il s'agit de comprendre une part de la subjectivation génocidaire et la façon dont les rapports de pouvoir qu'elle impulse passent par les corps. Nous rejoignons ainsi toute une tradition anthropologique qui, de Veena Das 17 à Joao Biehl 18 en passant par Arthur Kleinman ${ }^{19}$, a composé une

14. Crossley N., "Mapping reflexive body techniques: on body modification and maintenance ", Body E Society, vol. $11, \mathrm{n}^{\circ} 1,2005$, pp. 1-35.

15. Cité par Nkulikiyinka J-B., Introduction à la danse rwandaise traditionnelle, Tervuren, Annales des Sciences humaines, 2002, p. 166.

16. Pluriel de umuhamirizo.

17. Das V., Life and words: violence and the descent to the ordinary, Berkeley, University of California Press, 2007.

18. Biehl J., Vita: life in a zone of social abandonment, Berkeley, University of California Press, 2005.

19. Das V., Kleinman A., Ramphele M. (eds.), Violence and subjectivity, Berkeley, University of California Press, 2000. 
anthropologie des subjectivités largement inspirée des travaux du philosophe français. En matière de danse, nous nous rapprochons de même des travaux pionniers de J. Clyde Mitchell, qui considère la « situation dansée » non dans le cadre d'une fin en soi, mais plutôt comme un prisme autour duquel les conflictualités sociales se matérialisent ${ }^{20}$. Selon l'environnement social et politique de ces répertoires symboliques et technologiques, il existerait selon lui un espace de tension et d'opposition qui ne pourrait se relâcher que dans la violence, ou dans ce que Randall Collins appelle une "panique aggravée 21 ». Il s'agit là de la situation la plus dangereuse et la plus explosive qui soit ; situation dont les « techniques réflexives du corps » constituent, par hypothèse, les coulisses.

Au cour de la situation génocidaire que nous explorons se trouve une mise à disposition des corps par les techniques qu'ils mettent en mouvement et qu'ils s'approprient. À ce sujet, nous postulons que ces techniques pourraient - selon des circonstances sociales et politiques déterminantes - constituer une forme d'initiation ouvrant sur les pratiques de la violence de masse. Dans notre cas, il s'agit de comprendre comment - par le medium de la danse guerrière - se forment une hexis guerrière potentiellement mobilisable dans les pratiques, s'articulant aux imaginaires du génocide. Notre propos consiste ainsi à décrire et analyser la façon dont se tisse le lien entre la sédimentation d'une disposition agonistique et son activation dans la violence aussi extrême que paradoxalement normalisée du génocide. Pour ce faire, nous combinons à la littérature ethnographique et microsociologique sur le génocide, un corpus d'archives et de témoignages collectés en Belgique, en Italie et au Rwanda entre 2007 et 2014. Ces sources - archives missionnaires et gouvernementales, monographies, entretiens menés auprès d'anciens dirigeants rwandais et de plusieurs rescapés du génocide 22 - ont été analysées dans le souci de reconstituer les médiations par lesquelles une formation chorégraphique a été mobilisée dans les arènes de la violence de masse. Nous suggérons que dans la période visée (entre 1987 et 1994), les danses guerrières imibamirizo agencent des corps dont les parties (membres, fictions symboliques, répartitions matérielles) entrent en tension. Dans les conceptions que s'en font les acteurs et les

20. Mitchell J. C., The Kalela Dance. Aspects of Social Relationships among Urban Africans in Northern Rhodesia, Manchester, Manchester UP on behalf of the Rhodes Livingstone Institute, 1956.

21. Collins R., Violence. A Micro-Sociological Theory, Princeton \& Oxford, Princeton University Press, 2008.

22. Archives de la Société des missionnaires d'Afrique (ARCHPB), Rome : lettres de règle, rapports d'activités; Centre de documentation de la section d'ethnohistoire et d'ethnosociologie du Musée royal de l'Afrique centrale, Tervuren : monographies datant de la seconde république ; Archives nationales du Rwanda, Stade Amahoro, Kigali : coupures de presse, manifestes programmes, brochures ; Archives africaines du ministère des Affaires Étrangères, Bruxelles : rapports politiques ; Nous avons associé l'analyse de ces sources à huit entretiens menés en Belgique et au Rwanda au cours de l'année 2007 : quatre auprès de rescapés du massacre, quatre auprès d'anciens dirigeants de la seconde république (bourgmestres, préfets et ministres). 
techniques qu'ils s'approprient, ces corps contiendraient également leurs "impuretés » (leurs « virus », identifiés à l'infection du corps social par les Tutsi), à l'encontre desquels il va s'agir de prendre des mesure prophylactiques. Le démembrement des corps ne serait-il pas dès lors une manière de rétrécir l'action de ceux qui ne peuvent plus être membres du corps social ? On découpe ainsi les membres surnuméraires comme on rétablirait la santé d'un organisme purifié de ce qui semble l'infecter. Afin de mettre à l'épreuve cette dernière hypothèse, il s'agit d'explorer la part «dansée » de cette « mise en ordre » de la violence génocidaire, dans le souci de comprendre comment ces techniques se sont inscrites dans la matérialisation d'une idéologie qui a désigné ses victimes émissaires ${ }^{23}$.

\section{Faire fuir l'ennemi, purifier le « mal»}

Des années 1960 aux années 1980, la continuité des procédures d'élimination politique et physique des éléments « inappropriés » à l'ordre de la « révolution hutu »-soit les individus présentant le caractère d'une alliance potentielle avec les troupes de la diaspora tutsi - ne fait aucun doute. C'est ainsi que chaque situation de tension entraînée par la réintroduction potentielle des « ennemis de la démocratie » (ceux que les gouvernements du Rwanda dénomment les «féodaux ») génère - en retour - la mobilisation d'éléments hutu affectés à la purification ethnique d'un territoire donné. Ces formations radicalisées puisent leurs ressources matérielles et idéologiques au niveau local, au sein des communes et des préfectures les plus engagées dans le maintien de «l'ordre hutu» face au « danger tutsi ».

«C'était notre devoir de défendre la démocratie, donc le pouvoir du peuple! Alors quand il se passait des choses qui pouvaient la mettre en danger, il fallait résoudre la situation et éviter que les choses vacillent. Cela faisait partie du quotidien des dirigeants ! Moi à Kigali j'ai connu plusieurs fois des désordres. Alors on a essayé de calmer les choses, mais tous les gens ne tenaient pas forcément à les arranger [...]. Alors on a essayé de mobiliser les gens pour développer le pays sur de bonnes bases. Dans les communes il y avait des groupes de jeunes qui faisaient des travaux collectifs, d'autres qui chantaient, qui dansaient... C’est le président qui avait eu cette idée, et nous nous étions chargés d'organiser tout ça 24 ».

Sous la Seconde république rwandaise (1973-1994), les entités administratives décentralisées que constituaient les communes se composaient de plu-

23. Nous tenons à chaleureusement remercier Jérôme Beauchez, qui a réalisé une analyse critique passionnante de ce texte, analyse dont nous nous sommes inspirés tant dans la forme que dans le fond de notre propos.

24. André Sebatware (ancien bourgmestre, préfet, puis ministre du gouvernement Habyarimana), entretien réalisé à son domicile de Bruxelles, janvier 2007. 
sieurs secteurs (regroupant environ 5000 personnes) dans lesquels des responsables de cellules (regroupant environ 1000 personnes) étaient chargés d'exécuter les ordres du bourgmestre ${ }^{25}$. À partir de 1977, le parti unique MRND institua le fait que chaque cellule devait concevoir un dispositif « d'animation politique » composé d'une troupe de chant et de danse ${ }^{26}$. Le recrutement des membres était confié à des comités de cinq ou six personnes choisies, qui parcouraient les environs du centre afin d'affilier de nouveaux danseurs (agriculteurs, petits commerçants, ouvriers, jeunes délinquants) à ce programme de « développement culturel » 27.

Confiés à des spécialistes de danse guerrière, les entraînements avaient lieu au moins une fois par semaine. Chaque séance commençait par les déclamations des défis du jour (guhiga). Le ton était donné par l’animateur principal : «Il pouvait choisir par exemple nkubito, ce qui incite à la vaillance et à la rapidité. Alors on donnait ce thème et le ton de ce thème, et chacun devait dire ce qu'il allait faire 28 ». Dans le cadre des cellules communales d'animation, ces défis pouvaient s'articuler à la promesse d'un engagement sans faille en faveur de l'ordre développementaliste prôné par le gouvernement :

«Ils disaient qu'il allaient lutter pour leur pays, que nul ne saurait surpasser les héros du MRND, qu'ils ne craignaient pas la bataille, et qu'ils protègeraient le Rwanda contre tous ceux qui cherchaient à le combattre 29 ».

De fait, les défis des intore opérant dans les centres communaux rejoignaient la ligne d'auto-défense du régime vis à vis des «courants maléfiques » qui, selon les termes du MRND, pouvaient porter atteinte à la « nation rwandaise » et provoquer sa «désintégration » 30. L'imibigo (défi) est lancé. Il s'agit d'une préparation des corps à leur mise en action, couplée à des processus d'identification au «combat » que s'apprête à mener chaque intore : «Comment oses-tu me défier? Je suis le brave que nul ne saurait dépasser. Je suis l'expert qui ne cesse d'entasser l'adversaire dans des mares de sang figé 31 ».

25. Straton Semanyenzi, entretien réalisé à son domicile de Bruxelles, janvier 2007. Lire aussi Human Right Watch (HRW), Fédération internationale des droits de l'homme (FIDH), Aucun témoin ne doit survivre, Paris, Karthala, 1999, pp. 55-57.

26. L'ensemble des personnes que nous avons rencontrées dans le cadre de nos entretiens nous ont confirmé cette information. Voir sur le même sujet Kimonyo J-P., Rwanda. Un génocide populaire, op. cit., pp. 259-262.

27. Les anciens dirigeants que nous avons rencontrés s'accordent sur le fait de qualifier ce dispositif politique comme l'une des facettes d'un programme plus large de développement économique, social et culturel. Sur le rôle joué par des intermédiaires (social brokers) dans le passage de l'idéologie du développement à l'ordre du génocide, lire Emmanuel Viret, «La langue amère des temps nouveaux. Dynamiques de la violence au Rwanda rural », op. cit.

28. Entretien avec Léon Musoni, ancien danseur, Kigali, août 2007.

29. Jean-Damascène $X$, rescapé, interviewé à plusieurs reprises à Butare, juillet-août 2007.

30. Discours du président Habyarimana à l'ouverture du 4ème congrès ordinaire du MRND, le 26 juin 1993, extrait, Archives nationales du Rwanda, Kigali. 
Suite au guhiga, l'action guhamiriza se déploie selon une triple dimension symbolique, matérielle et politique. Vêtus de pagnes et de coiffes guerrières, accompagnés de lances, d'arcs ou de boucliers, les danseurs effectuent un enchaînement de sauts, de feintes, d'assauts ou d'esquives. La technique s'inscrit dans la construction de l'appareil collectif de conquête et de défense du territoire de la danse. Simultanément, une compétition s'exerce à l'intérieur du groupe, au centre de la lutte que mènent des « corps noirs » (impurs) face à des « corps blancs» (purs, fastes). Jean-Baptiste Nkulikiyinka a réalisé un schéma de formalisation de l'organisation matérielle, spatiale et symbolique de la chorégraphie. Dans l'agencement qu'il présente, les lignes de bataille ingamba forment les unités collectives de base du corps-dansant. À l'avant de la scène, la première rangée est composée des danseurs les plus compétents. Les suivantes sont considérées comme des lignes d'appoint. Les subdivisions de ces ensembles rendent compte d'une organisation spatiale codifiée. Chaque rangée de danseurs est répartie en trois parties principales : le milieu ou «nombril »; les deux flancs ou « coudes »; les extrémités ou « ailes » 32 .

Aile droite Coude droit Nombril Coude gauche Aile gauche

\begin{tabular}{|ll|ll|lll|ll|lll|}
\hline 0 & 0 & 0 & 0 & 0 & 0 & 0 & 0 & 0 & 0 & 0 \\
0 & 0 & 0 & 0 & & 0 & & 0 & 0 & & 0 & 0 \\
& & & & 0 & 0 & 0 & & & & & \\
& & & & & 0 & & & & & & \\
\hline
\end{tabular}

Imiyumbu

« Les Noirs »
Abanyamukondo

«Ceux du nombril »
Ibinyange

"Les Tout Blancs »

\section{Front de scène}

31. Charles Ndekwe, ancien danseur, rescapé, interviewé à la paroisse de Kimonyi au cours du mois d'août 2007.

32. Cette figure de la danse umuhamirizo a été réalisée à partir de celle que propose Nkulikiyinka J-B., dans le cadre d'une ethnographie historique des chorégraphies guerrières au Rwanda, op. cit., p. 194. 
Cet agencement est conçu comme un ordre hiérarchique matérialisé par les actions des danseurs. Elles sont en premier lieu associées aux rôles et aux places qu'ils occupent. À partir d'un centre composé des éléments les plus compétents, le corps de la danse se déploie du «nombril » jusqu'aux « ailes ». La danse prend les allures d'un corps en mouvement. Chaque individu compose un membre de cette entité, et chaque membre (ou partie du corps : ici les danseurs) fait partie d'un organe plus large (ici le « nombril », les « coudes » et les «ailes ») qui a sa fonction propre, différente des autres. Inégaux en puissance, en moyens et en attributions, ces organes sont complémentaires et se fondent dans un tout organique.

Au cœur du «nombril » se trouve le « capitaine », chargé de mener le jeu; les autres danseurs règlent leur prestation sur la sienne. Partant du centre du corps incarné par le «nombril », les mouvements des «coudes » transmettent la force physique et le fond sonore nécessaire à la cohérence de l'ensemble du corps. L'ensemble circule jusqu'aux extrémités, qui agitent leurs « ailes » dans le strict respect de la hiérarchie gestuelle et musicale orchestrée depuis le « nombril », transmise par les « coudes ». La hiérarchie se fonde donc sur un ordre agencé de l'avant vers l'arrière et du centre vers les extrémités. La différenciation qui s'opère entre la droite et la gauche est symboliquement caractérisée par les appellations « Noirs » (à droite) et «Tout Blancs » (à gauche). Ces deux groupes se livrent à une compétition permanente qui permet - in fine de désigner l'élément le plus « compétent » : celui qui répond le mieux au ton donné (vaillance, ardeur, rapidité, etc.) et qui porte plus de « coups » que ses concurrents à l'adversaire de l'action mobilisée.

Du début des années 1990 au mois de juillet 1994, le glissement de ces conduites chorégraphiques guerrières vers la mise en œuvre du génocide a été grandement conditionné par l'engagement (volontaire, conseillé ou forcé) de quelques milliers de danseurs dans les groupes extrémistes qui menèrent les pogroms (notamment les groupes interahamwe). À partir de l'année 1991 et conjointement à l'officialisation du multipartisme ${ }^{33}$, les territoires culturels des partis politiques en présence se radicalisent :

"Je savais tout simplement que j'étais membre du MDR, car celui qui se refusait d'adhérer à ce parti était condamné. C'était une façon de se racheter. [...] Donc il y avait des meetings, nous passions toute la journée à danser, celui qui le pouvait, celui qui n'était pas capable, il restait à la maison, mais avec sa carte, de façon que si on le surprenait, il pouvait montrer cette carte pour que les autres voient qu'il était lui aussi membre. C’est comme ça que nous vivions ${ }^{34}$ ».

33. Sous la Seconde République, le multipartisme fut instauré suite à l'amendement constitutionnel du 10 juin 1991. Il visait à reconnaître la quinzaine de partis politiques développés avant et après sa mise en application. 
Au cours de l'année 1992, ces espaces politico-culturels constituent l'un des viviers du recrutement de miliciens préparés à briser l'opposition politique par la violence. Dans le monde très concret de l'initiation au combat, il n'est plus question de danser; il s'agit de parfaire sa formation dans l'exercice des techniques et des objets appelés à constituer un front d'" auto-défense » face aux opposants potentiels au « développement » de l'institution : «En bref, nous les entrainions à courir longtemps et acquérir de l'endurance, à monter à l'aide d'une corde, à tuer avec le couteau et aussi aux exercices de tir 35 ». La guerre menée par d'anciens réfugiés tutsi depuis l'Ouganda et le Burundi voisins est un élément important de justification de cette initiation milicienne. Entre 1990 et 1993, chaque tentative d'incursion de l'Armée patriotique rwandaise (APR) est suivie de la mobilisation de groupes conditionnés à l'idée de "survie de l'ethnie majoritaire », qui massacrent des milliers de Tutsi identifiés aux «alliés de l'intérieur » du FPR ${ }^{36}$.

Dans ce cadre, et conjointement à la violence de masse qui s'exerce dans le champ matériel et idéologique des combats miliciens menés par le mouvement Hutu Power ${ }^{37}$, certains de ses membres participent encore (jusqu'en 1994) à des séances de danse guerrière. La combinaison de l'idéologie milicienne antiTutsi aux techniques de danse donne corps à la technologie politique du mouvement. Dans cette situation, des actions menées auprès des Tutsi de la cellule matérialisent le paradigme d'une opposition ethnique entre Hutu et Tutsi, révélant dans l'espace de la danse le « combat » plus large dans lequel les radicaux hutu se trouvent engagés. À l'échelle locale, il peut s'agir de faire danser de force (sous peine de coups et des heures durant) certains Tutsi de la zone, contraints d'ajuster leurs pas à des chants de louanges au parti politique dans lequel s'exerce l'action (comme par exemple celui des partisans du MDR 38 ). Un extrait de l'un de ces chants (le plus diffusé de l'époque) indique la férocité avec laquelle les danseurs hutu radicalisés allaient s'engager dans la technologie plus large du génocide :

"Qu'ils viennent les fils de Sebahinzi! Nous devons savoir que si les Inkotanyi prenaient le pouvoir par les armes, ce qui est d'ailleurs impossible, leurs différents partis disparaîtraient et leurs membres seraient exterminés comme ce fut le cas pour les roitelets hutu avant que le lendemain le Tutsi victorieux ne proclame : "Il était une fois,

34. Témoignage collecté et cité par Kimonyo J-P., Rwanda. Un génocide populaire, op. cit., p. 312 .

35. J*, ancien interahamwe, Témoignages filmés par Georges Kapler et discussion Journée du Mardi 23 mars, document de la Commission d'enquête citoyenne pour la vérité sur l'implication française dans le génocide des Tutsi, http://cec.rwanda.free.fr, 2004.

36. Le FPR prit le pouvoir en juillet 1994, suite à la guerre qu'il mena face aux troupes du gouvernement rwandais.

37. Terme qui désigne l'idéologie et les actions les plus radicales menées par les groupes politiques en présence.

38. Le parti de l'ancien président Kayibanda, au pouvoir de 1961 à 1973. 
que ce ne soit plus. Les chiens et les rats sont morts, seuls survivent la vache et le tambour. Nous ne nous souvenons pas vraiment de cet épisode" $39 »$.

Au début des années 1990, les chants des danseurs, comme les récits radiophoniques de leurs épopées, donnaient une tonalité émulatrice au monde matériel et imaginaire de l'auto-défense face aux « ennemis » de la République ${ }^{40}$. Ils reprenaient en ces termes le répertoire bien connu de "l'animation ", dans lequel s'inscrivait - depuis la fin des années 1970 - les danses guerrières imihamirizo. Mais de fait, comment pourrait-on dire qu'au cours des années 1980, un jeune paysan rwandais qui s'adonnait à une séance de danse guerrière se préparait à utiliser une lance pour décimer - au cours de l'année 1994 - ses voisins tutsi ? Il ne s’y préparait pas. Il s'exerçait à « faire fuir l'ennemi de sorte que celui-ci prenne ses jambes à son cou sans oser même se retourner».

\section{Danser la guerre, exterminer les Tutsi}

Au cœur des espaces sociaux et politiques dédiés aux chorégraphies guerrières, l'ordre des danseurs n'est pas celui du génocide. Pourtant et sous de multiples facettes, il le rappelle. Dans le cadre d'une symbolique plus ancienne, le corps rwandais est appelé à temporiser la situation ambivalente d'un monde terrestre où la mort ne cesse de guetter la vie ${ }^{41}$. Cette conception désigne les «corps blancs " comme des éléments fastes, vivants, alors que les « corps noirs » manifestent l'oubli, la disparition de la surface des vivants. Suivant le schéma précédent, alors que les «Noirs » et les «Tout Blancs» déploient - dans une relation concurrentielle - des forces obscures d'un côté, fastes de l'autre, la convergence de ces fluides vers le centre du corps assure un rééquilibrage de l'apparente opposition pur/impur, vice/vertu. Si les « Noirs » incarnent le « mal » et les «Blancs » le « bien », la lutte qu'ils mènent doit conduire à la victoire d'une seule et même entité : l'ordre de la guerre menée par le mouvement gubamiriza. Au cour de cet «ordre » se trouve le combat du centre : axe de l'exercice légitime du pouvoir incarné par l'action des danseurs.

Il s'agit d'un appareil de production des techniques du «bien » et du « mal », des vices et vertus par le médium desquelles se forge la normativité du danseur guerrier : où l'oisiveté se décline en ardeur ; où la crainte renvoie à l'audace; où le désordre s'institue en ordre. L'ennemi est donc en premier lieu celui qui s'oppose à l'organisation, qui tente de la déstabiliser, allant jusqu'au

39. Chrétien J-P. (dir.), Rwanda. Les médias du génocide, op. cit., pp. 353-354.

40. Les rescapés que nous avons interviewés vont même jusqu’à affirmer le ton humoristique que pouvaient prendre les appels à la traque des Tutsi, ainsi que la joie des miliciens lorsqu'ils avaient accompli leur « mission ", partageant bières et marijuana au rythme des chants et des récits de leur journée de «travail ». 
combat final. Ce dernier se trouve associé à la subjectivation d'un certain nombre de ressources pratiques et symboliques : porter et recevoir un coup ; s'attendre à être déstabilisé ; armer son corps ; connaître les manifestations du faste et de l'impur. Dans l'ensemble, les membres combattent les forces d'opposition au modèle légitime forgé par l'institution. Le pouvoir qu'elle exerce s'apparente au produit disséminé de « l'anatomie politique » du dispositif. Dans les arènes normatives $\mathrm{du}$ « bien » et $\mathrm{du}$ « mal » comme dans les techniques belliqueuses menées par la troupe, il donne un sens social à l'expérience du combat. La technologie de l'ordre guerrier ainsi créé laisse apparaître l'ennemi de son développement : les corps infectés par un ensemble de virus pouvant porter atteinte à l'intégrité des «sauveurs ». Chacun lutte, en interne et dans la projection du groupe face à l'extérieur, contre la dissémination des « maladies » qui pourraient atteindre le groupe. Et chacun renforce, à titre individuel et collectif, la lutte engagée face au désordre, à l'impur, à l'irrégularité.

Sous la Seconde république rwandaise, un tel dispositif pratique et symbolique a alimenté les fractures autour desquelles se sont agencés les territoires de l'institution et les ennemis de l'ordre subjectivé par les techniques de danse. Depuis 1990, la guerre enclenchée entre le pouvoir rwandais et les armées du FPR alimentait l'angoisse paranoïaque des membres de mouvements politiques et (para)militaires préparés à systématiquement riposter aux attaques du FPR par le massacre de leurs «alliés ». Conjointement, et notamment à partir de 1991, les cellules de danse affirment de nouveaux besoins : il s'agit de collaborer avec les partis politiques en présence (MDR, MRND, etc.) et d'offrir aux Hutu de la région la possibilité de remporter la « guerre » 42 que ces partis entendent mener face aux « ennemis de la nation» (les Tutsi) ${ }^{43}$. À partir de ce moment, les quelques Tutsi qui officient encore dans les troupes communales sont considérés comme des "maladies à soigner». Il s'agit de prendre à leur encontre des mesures prophylactiques qui se manifestent dans la répétition de danses et de chants pour «la sécurité de l'État »:

«Dis aux fils de Sebahinzi de venir! Ils doivent comprendre que le peuple majoritaire doit s'unir et avoir un seul objectif pour sauvegarder ses intérêts. Les fils de Sebahinzi doivent soutenir fermement, avec abnégation les forces armées et au besoin les rejoindre pour protéger la mère patrie et l’intégrité de la République ${ }^{44}$ ».

41. Lire à ce sujet Smith P., «Aspects de l'esthétique au Rwanda », L’Homme, vol. 25, n 96, 1985, pp. 7-22.

42. Il s'agit du terme employé par les quatre anciens dirigeants que nous avons rencontrés, qui rejoint les imaginaires guerriers articulés à la mise en œuvre du massacre.

43. Projet qui préexistait à l'avènement du multipartisme, et qui fut exprimé dès l'essor du parti unique MRND : MRND, Manifeste et Statuts, Archives nationales du Rwanda, Kigali.

44. Chrétien J-P. (dir.), Rwanda. Les médias du génocide, op. cit., pp. 353-354. 
Le 6 avril 1994, des milliers de militaires, miliciens et civils apprennent la mort du président Habyarimana ${ }^{45}$. Associé aux violentes rivalités entre les partis politiques en présence, à la crainte et à la haine du régime envers ceux qu'il identifie aux « féodaux de l'extérieur et de l'intérieur » (soit le mouvement du FPR et les Tutsi du pays), la faille politique causée par l'assassinat du président est un appel à la conquête du pouvoir. Des groupes d'intermédiaires locaux (préfets, bourgmestres, militaires, responsables de mouvements de jeunesses, chefs de groupes miliciens) activent des technologies politiques de mise en œuvre de la violence collective. Le principal vivier de recrutement des milices du génocide est composé des membres les plus actifs de nombreuses organisations : supporters et joueurs de football, danseurs-guerriers, jeunes et adultes militants politiques, membres de groupes (para)militaires potentiellement mobilisables par le régime ("l'armée populaire " convoquée par Monsieur Z 46 dès janvier 1993) ${ }^{47}$. Ces organisations composent les unités paraétatiques autour desquelles se tisse peu à peu un lien entre les dispositions agonistiques des danseurs et les technologies politiques plus larges du corps génocidaire. Un tel « lien » peut s'expliquer par le fait que les danses guerrières agissent selon une triple dimension psychique, symbolique et sensorielle. Depuis les chorégraphies menées dans les cellules communales de l'intérieur $\mathrm{du}$ Rwanda, la mise en tension des danseurs se renforce sous la menace de voir les Tutsi piétiner leurs territoires. Les techniques des initiés hutu peuvent se lire face à ceux qu'ils considèrent comme leurs ennemis les plus intimes ${ }^{48}$. La «guerre des frontières » s'actualise sous les pas et les chants des troupes:

« Nous partons en guerre. Nous répondons à l'appel des frontières. Nous voici, pareils au fer de lance, ennemis déclarés de la peur [...]. Réponds à cet appel à la guerre, toi, lance prête à s'élancer. Manie ta lance, pare avec ton bouclier, dresse les flèches dans ton arc et déclame tes hauts faits à l'agrément de tous, tant que nous sommes 49 ».

À l'intérieur du pays, les danseurs-guerriers hutu les plus radicalisés ont désigné les victimes émissaires de leur combat (les Tutsi) dans ce que l'on peut appeler une «lutte pour la survie de la troupe »: les imaginaires d'autodéfense qui se déploient dans les gestes et paroles des danseurs constituent la trame symbolique la plus aggravante du phénomène ; cédant à une sorte de

45. Soit l'attentat contre l'avion présidentiel du 6 avril 1994, qui déclencha le commencement des pogroms de masse à l'encontre des Tutsi.

46. Soit Protais Zigiranyirazo, considéré comme l'un des principaux cerveaux du génocide.

47. Lire à ce sujet Riot T., "Les politique de loisir et le génocide des Rwandais tutsi. Du racisme culturel aux donjons de la mémoire ", Politique africaine, n ${ }^{\circ} 33,2014$, pp. 131-151.

48. Dans un tout autre contexte, mais selon une situation d'intimité meurtrière comparable, lire Theidon K., Intimate Enemies. Violence and Reconciliation in Peru, Philadelphia, University of Pennsylvania Press, 2013.

49. Chant retranscrit par Rugamba C., Chansons rwandaises, Butare, Institut national de la recherche scientifique, 1979, pp. 219-222. 
«panique aggravée » face au « risque » d'être littéralement « mangés » par les Tutsi, des danseurs hutu prennent les chemins d'une radicalisation politique des plus extrêmes : "Certains étaient devenus fous... Alors ils ont arrêté de danser, et se sont mis au travail $50 »$.

Au moment de l'exécution du génocide, la rationalité du massacre transparaît de l'action collective et individuelle des tueurs. Elle consiste dans un premier temps à encercler les futures victimes, et à placer les cibles dans la visée de la première ligne. Composée de forces militaires et policières, elle mitraille les premiers réfugiés à sa portée. La seconde ligne de miliciens armés de fusils et de machettes effectue l'essentiel du «travail ». On reconnaît d'anciens membres et cadres des cellules artisanales et d'animation de la commune ${ }^{51}$. Cruauté et efficacité s'imbriquent dans les techniques qu'ils utilisent pour mettre fin à la « race tutsi ». Tandis que certains découpent les tibias et les avants bras de ceux qu'ils dénomment les inyenzi, d'autres s'emploient à ensevelir les corps encore vivants de leurs victimes. La dernière ligne a pour rôle et mission de terminer le «travail ». Elle y associe saignées, diffraction des corps, défigurations. Ce qui n’est pas « croyable » devient raison, animé par des techniques de massacre précises. Mais ces techniques ne sont pas uniquement déterminées par la rationalité de leur mise en œuvre. Du point de vue du psychisme des tueurs, l'angoisse de se trouver confronter au "virus » tutsi est l'un des préalables à la mutilation de leur corps.

Les actions de découpe des membres (notamment les tibias et les bras) ont ainsi d'autres significations que la seule cruauté. Dans l'espace particulier de la danse, ces actions prophylactiques (coups, traques, etc.) ne donnent pas lieu à des mesures d'élimination concrète des Tutsi. Ces dernières se réalisent suite au transfert de ces "syndromes » au sein des organisations paramilitaires affectées à l'exécution des pogroms. Entre ces deux espaces (les cellules de danse guerrière et les formations miliciennes) circulent les pratiques et les imaginaires formés et réactualisés d'une organisation à l'autre. Au sein des groupes miliciens, l'apprentissage du maniement des armes n'est qu'une dimension d'un faisceau bien plus large d'éléments psychologiques, imaginaires et matériels plus anciens. Les dispositions à la guerre totale acquises par les miliciens se lient aux imaginaires dévorants de "l'ogre tutsi ». Les techniques qu'ils ont incorporées dans les cellules culturelles et politiques des communes leur donnent la possibilité de physiquement répondre au danger qu'ils ressentent : traques, feintes, attaques, jusque dans l'élimination systématique de l'ensemble de leurs victimes émissaires.

50. Charles Ndekwe, op. cit.

51. Nous prenons ici appui sur les témoignages que nous avons recueillis, ainsi que sur le travail de terrain mené par les équipes d'HRW et de la FIDH, Aucun témoin ne doit survivre..., op. cit. 
Mais de fait, les cibles visées par les milices extrémistes hutu se confondent dans la désignation d'un seul ennemi : le Tutsi, désigné comme le plus intime responsable des «maux» des Hutu. Dans une certaine mesure, les corps des tueurs ne font aussi plus qu'un : entité psycho-socio-biologique chargée de purifier le Rwanda du virus tutsi. Concrètement, il s'agit de couper tout ce qui dépasse, et en particulier les bras et les jambes, conduisant aux pires mutilations. C'est ici que dans une certaine mesure, la situation dansée qui consistait à purifier l'espace chorégraphique de ses ennemis potentiels donne matière à penser à la technologie du massacre. D'un espace à l'autre - et selon une mise en ouvre aggravante -, il s'agit de maintenir l'ordre du dispositif et de garantir la «santé » de ces membres; situation dans laquelle les membres surnuméraires potentiellement infectés doivent être éliminés. Les danseurs radicalisés et les miliciens hutu conçoivent ce « surnombre » dans l'anatomie politique du Tutsi, composée de bras et de jambes dont la taille leur rappelle la grandeur dévastatrice de «l'esprit féodal » qui ronge leurs affects. Dans la situation dansée comme dans le conflit armé et sociétal plus large qui gagne la subjectivité des tueurs, leur environnement est systématiquement repensé à la lumière d'une infection incarnée par «ce qui dépasse » du corps tutsi et qui pourrait conduire à la contamination de leur chair. Dans l'espace de la danse, il s'agit de réduire l'action des membres Tutsi par une plus forte mise en tension des techniques du groupe identifié à la «pureté » de l'ordre hutu. Dans l'espace du génocide, il s'agit de couper le mal à sa racine et de mutiler tout membre menaçant la "survie de l'espèce ». Des techniques de danse à celles des pogroms, l'on trouve ainsi quelque chose à penser dans l'ordre de l'activation d'une hexis guerrière purificatrice dont la subjectivation précède la mise en œuvre du massacre. Cette hexis se matérialise dans une séparation radicale des corps, alors même qu'ils étaient censés faire partie de cet ensemble organique qu'aurait pu figurer l'État rwandais. Or, dans les situations dansées, culturelles et politiques qui ont précédé le génocide, on constate que les frontières ethniques et symboliques s'instillent jusque dans la chair des auteurs des crimes et des victimes. Entre bien d'autres techniques réflexives du corps, les danses guerrières matérialisent ces frontières, les font apparaitre et les rendent palpables par le medium du combat imaginé qu'elles incarnent.

\section{Les corps de la violence génocidaire}

Dans la situation génocidaire, l'atrocité des tueries ne voile pas la rationalité de leur exercice. Elle présente l'univers pratique et symbolique qui précède l'activation des tueries. Un rescapé le formule en ces termes : «Ils ne coupaient par hasard, ils savaient s'y prendre et avaient leurs techniques $52 »$. Un ancien bourgmestre dénomme la mise en œuvre de cette action comme une « intention ${ }^{53}$. Elle fut pour partie conditionnée par la subjectivation de savoirs et

52. Jean-Damascène X., op. cit.

53. Straton Semanyenzi, op. cit. 
de techniques mobilisés par les unités affectées à l'exécution des pogroms et des mutilations corporelles qui les accompagnent.

Dans le cadre d'une conception plus ancienne du corps au Rwanda, les actions souvent réductrices et parfois mutilatrices exercées sur les membres et les organes des défunts consistaient à les rendre inaptes à revenir menacer l'activité de ceux qui, pour un temps, demeuraient aux frontières de la vie et de l'oubli ${ }^{54}$. Les techniques pouvaient consister à plier le corps, noircir sa peau, ou encore ligaturer ses articulations. Leur efficacité consistait en une protection face aux sources d'infection. Pour le mouvement extrémiste hutu et ses membres «danseurs », le corps tutsi est un « corps-pot». Les actes de manducation de la chair des vaincus (identifiés à la majorité raciale hutu) auraient nourri sa vigueur légendaire; ses organes seraient imprégnés de la putréfaction de ses victimes; sa peau, son odeur et ses fluides sont pour les tueurs autant d'indices de la mort incarnée par cet homme devenu un " animal démoniaque ». Sa figure et ses actes sont régulièrement identifiés au Kalinga, l'un des tambours royaux de l'ancienne monarchie Nyiginya. Pour les génocidaires, les guerres menées par le corps inyenzi ont forgé la matière du récipient (le Kalinga).

Ainsi, la principale tâche du corps tutsi aurait été d'apporter «vie » et nourriture à l'un des symboles de la fertilité guerrière du royaume. Les récits concordent pour formuler le fait qu'au retour des champs de bataille, les guerriers pouvaient accrocher les testicules des hommes de pouvoir qu'ils avaient vaincus. Le discours racialo-politique hutu a fait de ces «épopées » des éléments essentiels de son œuvre de reconstitution historique du Rwanda précolonial. Des données criminologiques, militaires ou encore psychologiques se sont formées à partir du cumul d'un savoir d'État caractérisant les Tutsi par les marques raciales et politiques qui ont dessiné plusieurs aspects de leur "irrégularité »: culture de la dissimulation, gloutonnerie, barbarie, etc. ${ }^{55}$ Dans ces corpus de connaissances qui nourrirent nombre de membres de l'animation politique ${ }^{56}$, le corps hutu est identifié à la principale cible des conquêtes tutsi ; les récits en font la substance essentielle des « repas »du Kalinga, ventre de la domination « hamite ${ }^{57}$. Les organes du pouvoir tutsi

54. Voir Hertefelt M. et Coupez A., La Royauté sacrée de l'ancien Rwanda : texte, traduction et commentaire de son rituel, Tervuren, Musée royal de l'Afrique centrale, 1964.

55. Dont la formalisation remonte à la période de décolonisation du pays, en ceci qu'elle postule une lutte atavique entre un peuple de défricheurs (hutu) et une caste de seigneurs (tutsi). Nous nous référons ici aux archives politiques de la période, et notamment au document Rapport de la 15ème session du Conseil Supérieur du Pays, du 19 mai au 29 mai 1958, Annexe 1 Exposé des représentants Bahutu et certains autres Bahutu, Archives du Ministère des Affaires Étrangères, RA/RU 11, Bruxelles.

56. Voir notamment la thèse de doctorat remaniée en pamphlet historique de l'un des principaux idéologues du génocide : Nahimana F., Le Rwanda. Émergence d'un État, Paris, L'Harmattan, 1993.

57. L'idéologie hamitique est née pendant l'époque coloniale. Elle propose de resituer l'ascendance des Tutsi de la région des Grands lacs dans la lignée raciale de populations originaires 
auraient été alimentés par l'énergie vitale des "défricheurs », devenus cibles d'une gloutonnerie physique et politique. Comme source d'épanouissement de la domination tutsi, la manducation des organes hutu aurait entrâné leur putréfaction : perspective dans laquelle le corps tutsi est un organe fétide constitué de la souillure de ses actes barbares. Si telle est la composition imaginée du corps tutsi et de son « aliment » hutu, l'action des génocidaires peut aussi se lire selon une mythologie politique appelée à rendre possible la « libération » du corps hutu des entrailles de son ogre.

L'objet de cette contribution (soit la sédimentation d'une disposition agonistique véhiculée par la danse dans l'ordre du génocide) nous a poussé à différencier les techniques chorégraphiques de celles qui ont directement gouverné l'action génocidaire. De notre point de vue, la subjectivation des techniques de danse s'inscrit au cœur d'une organisation sociale et politique (celle de «l'animation») dans laquelle des imaginaires «coupants» et des actions guerrières réorientent et structurent la réalité des initiés de façon durable. La disposition que nous évoquons s'inscrit dans un tel processus, autour duquel un certain entrainement martial des corps peut être mobilisé dans la pratique de la guerre, jusque dans les actes les plus extrêmes. Selon une telle dynamique, la supposée fonction cathartique des pratiques artistiques ne tient pas. Dans le cas rwandais, ces dernières officient en effet bien plus dans le cadre d'une «zone de tension » dont les membres entrent en relation d'opposition au sein du méta-organisme que compose le collectif des danseurs. Dans la situation sociale et politique que connut le pays au cours des années 1980-90, cette tension a trouvé son "relâchement » dans l'exercice de la violence de masse à l'encontre des Tutsi du pays. Pour le sociologue susceptible d'observer cette «montée en panique » 58 , il s'agit de la situation sociale la plus dangereuse et la plus explosive qui soit ; une situation - et son horreur - dont les danses guerrières constituent une part des coulisses étrangement rendues publiques.

de la corne de l'Afrique. Lire à ce sujet Chrétien J-P. et Kabanda M., Rwanda. Racisme et génocide. L'idéologie hamitique, Belin, Paris, 2013.

58. Collins R., Violence. A Micro-Sociological Theory, op. cit. 